\title{
Vibration Sensors Using Power Generation with Electret for Bridge Monitoring
}

\author{
Eiichi Sasaki, ${ }^{*}$ Takashi Ueda, Koichi Takeya, and Hiroshi Yamaguchi \\ Graduate School of Science and Engineering, Tokyo Institute of Technology, Meguro, Tokyo 152-8550, Japan
}

(Received August 11, 2016; accepted December 19, 2016)

Keywords: bridge, vibration, power generation, electret

The structural monitoring of civil engineering structures such as bridges has been of importance recently, and one of the key issues in structural monitoring is to grasp the dynamic behaviors of the structures. In this study, by utilizing the power generation characteristics of electrets, new innovative vibration sensors are investigated using electret material. The concept and the fundamental characteristics of the power generation sensors were described. Furthermore, the development of a new very low resonance frequency vibration sensor with electrets was undertaken, and the performance of the new sensors was evaluated.

\section{Introduction}

The structural monitoring of civil engineering structures has recently received attention. For bridge structures, the purposes of structural monitoring can be broadly classified into two: normal continuous monitoring to grasp and predict deterioration, ${ }^{(1-3)}$ and detection of anomalies due to extraordinary loads such as earthquakes and strong winds or due to aging. From the previous studies, it is known that to detect anomalies in bridge structures, the evaluation of changes in vibration characteristics, such as frequency and damping, is effective, as well as the detection of changes in the shapes of components as a result of deformation. ${ }^{(4-8)}$

For the monitoring system, wireless sensor technologies ${ }^{(6,9,10)}$ have been adopted to reduce the installation time and cost in the deployment of the system. Wireless sensor systems enable us to build a long-term health monitoring system for bridges more easily. In vibration measurements using wireless monitoring systems, MEMS acceleration sensors are normally used considering size and cost. ${ }^{(6,10)}$ In order to obtain the deformation shapes of structural components, conventional strain measurements and displacement measurements have been conducted, and for wireless monitoring systems, methods of conversion from acceleration to displacement have been investigated. ${ }^{(11,12)}$ However, MEMS acceleration sensors can include noise in data, and this causes large calculation errors in the double integration of acceleration for its conversion to displacement, so it is difficult to obtain accurate information on displacement in many cases. The required properties of MEMS acceleration sensors to obtain the displacement information of bridge structures have also been investigated. ${ }^{(12)}$ In addition, in wireless monitoring systems, power supply is a problem for long-term measurements. Energy harvesting, such as by using solar cells, is one of the promising solutions to securing a power supply for rechargeable batteries in the wireless

*Corresponding author: e-mail: sasaki.e.ab@m.titech.ac.jp http://dx.doi.org/10.18494/SAM.2017.1450 
monitoring systems, but the development of a new sensor suitable for long-term measurements with low energy consumption can also be a solution to the power problem.

In this study, we focus on energy-harvesting technology to change the vibration energy to electrical energy in developing new sensors. The energy harvesting using bridge vibration has been investigated for the purpose of power generation to support wireless measurement systems. ${ }^{(13-18)}$ On the other hand, in power generation using vibration, the generated electric power theoretically correlates with external disturbances, and so, conversely, it is possible to develop a sensor to evaluate external disturbances from generated electric power. Furthermore, as the sensor utilizes the electric power generation phenomenon, the sensor itself can be self-sufficient in electricity without the need for an external power supply, and thus can obtain data passively.

Among the various types of energy-harvesting devices, devices using electrets ${ }^{(16,17)}$ have some advantages for long-term monitoring systems. They are as small as other MEMS sensors and show long-term stability in power generation. ${ }^{(19)}$ Moreover, there is a possibility that the generated power is strongly related to the vibration velocity at the measured point as an external disturbance and that velocity can be evaluated from the generated power. It will enable us to obtain more accurate information on the displacement of structural components, because only single integration is needed to obtain the displacement from a velocity time series.

Previously developed energy harvesters using a vibrating electret have the comb-type structures to enhance the efficiency of power generation and also have higher resonance frequency than the dominant frequency level of bridge structures. Therefore, a simple structure of the electret electrode was proposed to elucidate the relationship between the vibration velocity and the generated power by numerical analysis, and a prototype sensor considering a relatively low frequency region was developed. ${ }^{(18)}$ In this study, to further investigate the proposed sensor, the idea and basic characteristics of the sensor are reviewed, and newly developed prototype sensors with different resonance frequencies were investigated.

\section{Proposed Electret Electrode Structure}

\subsection{Conventional electret electrode structure and power generation mechanism}

Figure 1 shows the electret energy-harvesting device with a conventional comb-type electret electrode structure. To enhance the power generation efficiency, the energy harvesting device has a comb-type structure. The size of the device is $20 \mathrm{~mm}$ by $20 \mathrm{~mm}$. The electret electrode

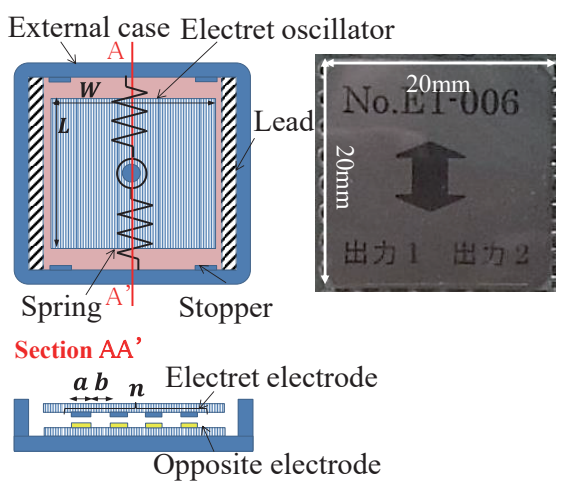

Fig. 1. (Color online) Structure of the energy harvester with electret. 
connected to springs faces the opposite metal electrode in the device. The electret material is always negatively charged, and the negative electrical charge causes a positive electrical charge on the opposite metal electrode via electrostatic induction. ${ }^{(17)}$ External vibration induces the vibration of the electret electrode connected to springs inside the energy harvesting device. The vibration of the electret electrode changes the facing area of the opposite metal electrode and causes electric potential differences and electric current flow. In the measurement system for the electret energyharvesting devices, constant resistance is used to measure the generated electricity as volts.

In Fig. 2(a), the typical generated power time series expressed in volts is shown. The generated power time series was measured under external vibration shown in Fig. 2(b). While the vibration displacement draws smooth sinusoidal curves, the generated voltage shows a ripple form. Therefore, a different simple electrode structure is appropriate for vibration measurements.

\subsection{Numerical simulation of conventional electret electrode structure}

In order to clarify the factors affecting the time series form of generated power expressed in volts, the power generation behaviors of the electret energy-harvesting device with a conventional electrode structure were investigated by numerical simulation. The simulation model consists of two basic parts, ${ }^{(20)}$ i.e., a mechanical part and an electrode part, as shown in Fig. 3. The vibration displacement of the electret electrode is calculated in the mechanical part, and the electrode part simulates the electric power generation using the calculated displacement of the electret electrode. In the mechanical part, the seismo-system model is used. The electret electrode is modelled as a

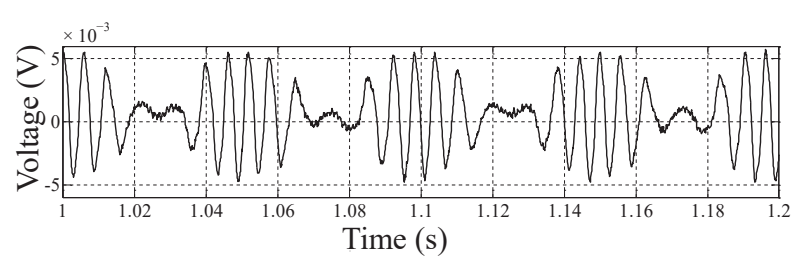

(a)

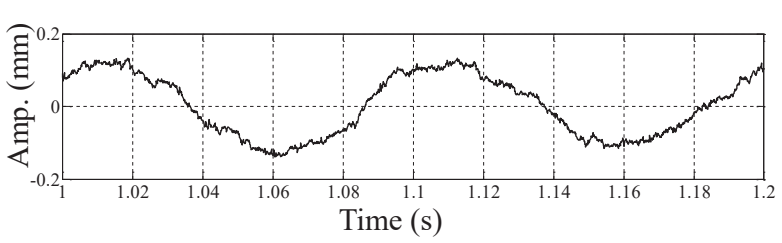

(b)

Fig. 2. Waveforms of generated voltage and vibration displacement. (a) Generated voltage. (b) Vibration displacement.

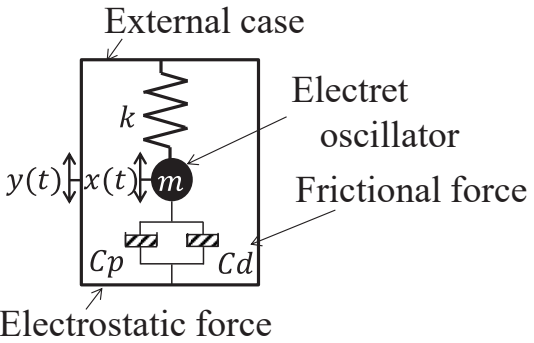

(a)

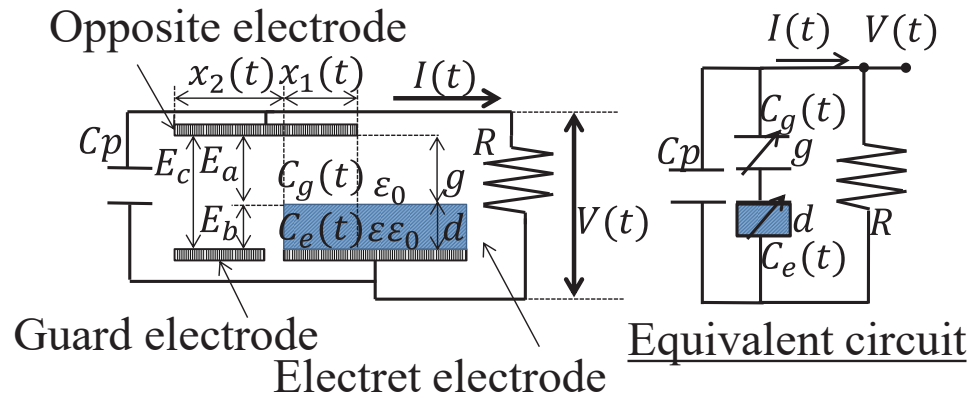

(b)

Fig. 3. (Color online) Numerical simulation model. (a) Model of mechanical part. (b) Model of electrode part. 
vibrator, and electrostatic force and friction force are considered for damping. The electrode part is modelled as a variable capacitor circuit.

In Table 1, the parameters used in the numerical simulation are shown. The parameter values were determined from the actual conditions of the device. In this study, parasitic capacitance was ignored because the measured value in the device was smaller than $2 \mathrm{pF}$. The simulation in the electrode part was performed considering only the power generation when the vibrator displacement is directly applied.

Figure 4 shows the voltage time series obtained in the vibration test of the electret power generation device and by simulation. The simulation was carried out under the same conditions as those in the test. The simulation result agrees well with the vibration test result. This means that the simulation can be used to predict the performance of a device.

In order to investigate the power generation processes in more detail, the electret electrode with only one comb was analyzed. Figure 5 shows the simulation results of electrode displacement, generated electric power expressed in volts, and vibration velocity. At the moments when the electret electrode crosses the opposite metal electrode, the generated voltage waveform becomes discontinuous and turns to the opposite phase. From the numerical simulation, it was observed that to understand the correlation between the generated electricity power and the vibration velocity, the crossing of the electret electrode over the opposite metal electrode must be prevented.

\subsection{Proposed electret electrode structure as vibration sensor}

Considering the abovementioned results, a new electret electrode structure for the vibration sensor, shown in Fig. 6, was proposed. To eliminate the crossing of the electret electrode over the opposite metal electrode and to keep the electret electrode always facing the opposite

Table 1

Parameters used in numerical simulation.

(a) Parameters of electrode part

\begin{tabular}{lc}
\hline Gap between electrodes $(\mu \mathrm{m})$ & 70 \\
Thickness of electrets $(\mu \mathrm{m})$ & 15 \\
Relative permittivity & 2.1 \\
Resistance $(\mathrm{M} \Omega)$ & 15 \\
Charge density of surface $\left(\mathrm{C} / \mathrm{m}^{2}\right)$ & $0.7 \times 10^{-3}$ \\
\hline (b) Dimensions of the device & \\
\hline Width of electret oscillator $(\mathrm{m})$ & 0.018 \\
Length of electret oscillator $(\mathrm{m})$ & 0.01 \\
Width of electret electrode $(\mu \mathrm{m})$ & 325 \\
Gap between electret electrodes $(\mu \mathrm{m})$ & 325 \\
Number of electret electrodes & 17
\end{tabular}

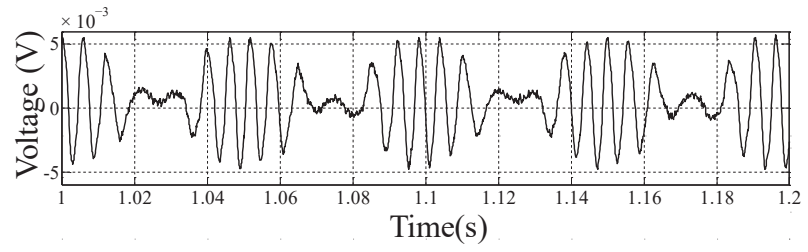

(a)

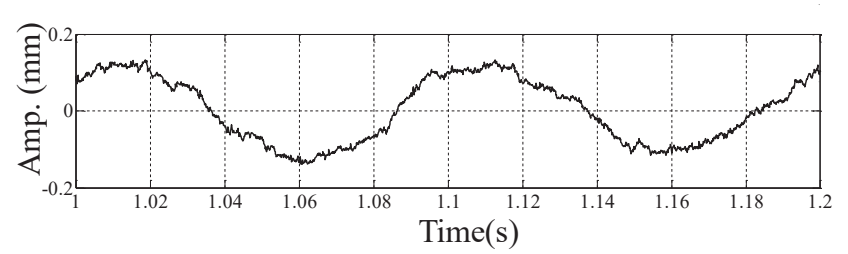

(b)

Fig. 4. Actual and simulated waveforms. (a) Actual waveform. (b) Simulated waveform. 


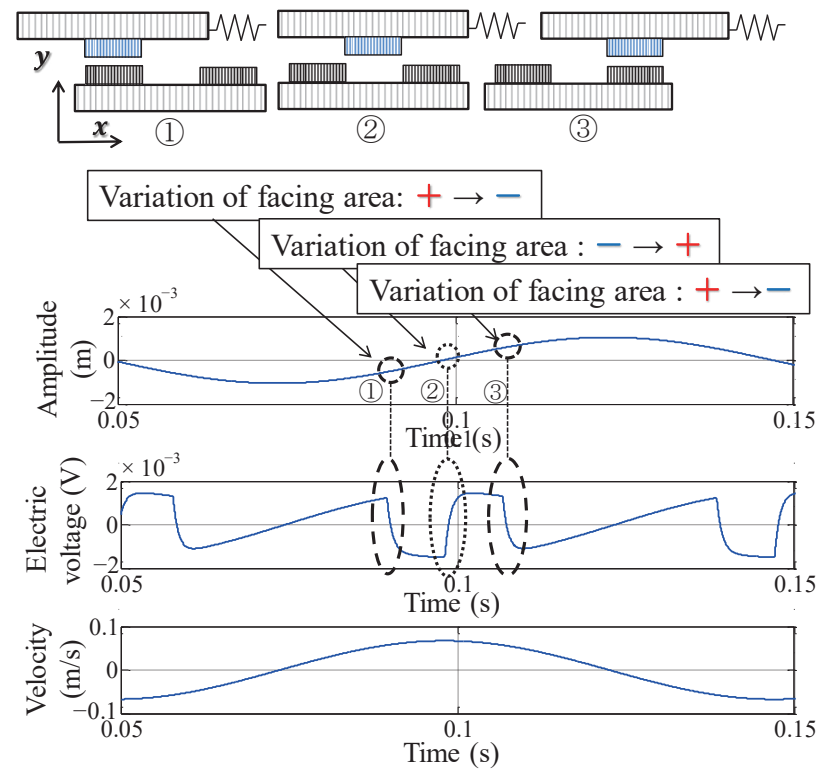

Fig. 5. (Color online) Analysis of waveforms by numerical simulation.

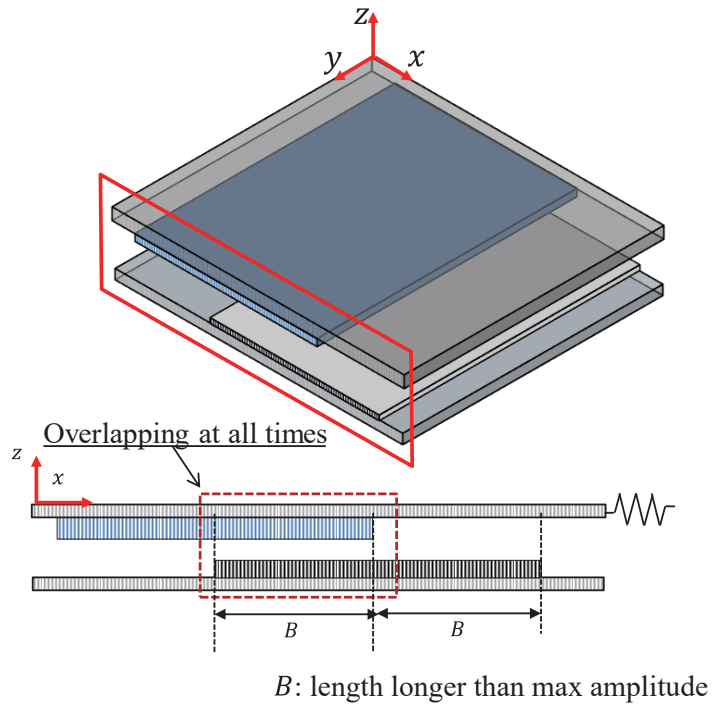

Fig. 6. (Color online) Proposed electret electrode structure.

metal electrode, a simple structure is considered. Only one electrode is used and the vibration displacement is limited to half the electrode length. It is expected that this electret electrode structure will cause no discontinuities in the generated voltage waveform. Figure 7 shows the simulation result of the proposed electret electrode structure. Figures 7(a) and 7(b) show the generated power shown in volts and the vibration velocity, respectively. The two waveforms are smooth and correspond well to each other. As shown in Fig. 7(c), the two waveforms are in the same phase. From these results, it was confirmed that the proposed electret electrode structure is appropriate for determining the vibration velocity from the generated power.

The proposed electret electrode structure is for measuring the vibration of bridge structures, and thus the generated power will be used mainly to analyze the vibration characteristics, not for energy harvesting. In the measurement system, the power supply will be prepared using batteries or other ways.

\section{Fundamental Characteristics of a Vibration Sensor Prototype}

A vibration sensor with the proposed electret electrode was actually manufactured. The shape and dimensions of the sensor are shown in Fig. 8. The parameters used in the sensor are listed in Table 2. The resonance frequency of the sensor is determined to be less than $10 \mathrm{~Hz}$ considering that the dominant frequency of typical bridge structures is on the order of $1 \mathrm{~Hz}$, which is lower than that of a previously developed energy harvesting device with an electret. The developed sensor consists of electrodes and springs, similarly to the electret energy-harvesting device shown in Fig. 1. The generated power in the sensor can be measured by connecting the two terminals of the sensor (see Fig. 8) to a measurement system as volts. In this section, the fundamental test results of the developed sensor are described. 


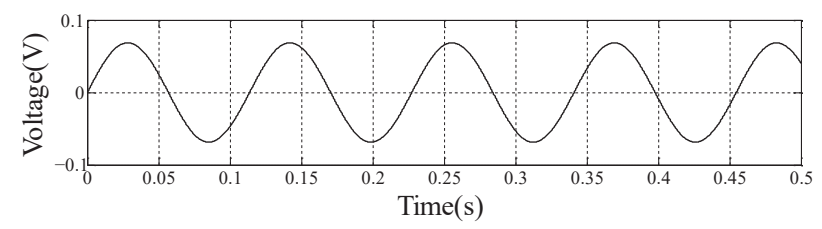

(a)

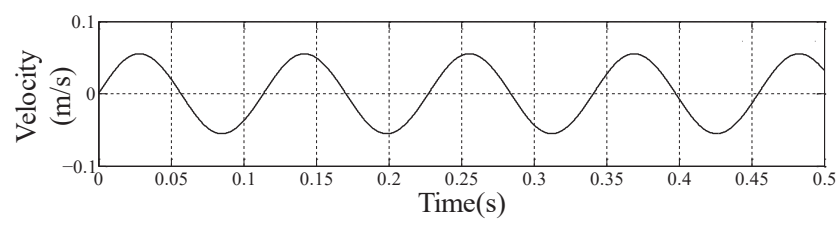

(b)

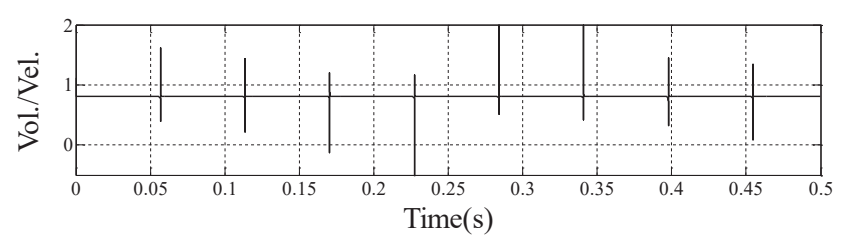

(c)

Fig. 7. Numerical simulation results for the proposed sensor. (a) Generated voltage. (b) Vibration velocity. (c) Ratio of generated voltage to vibration velocity.

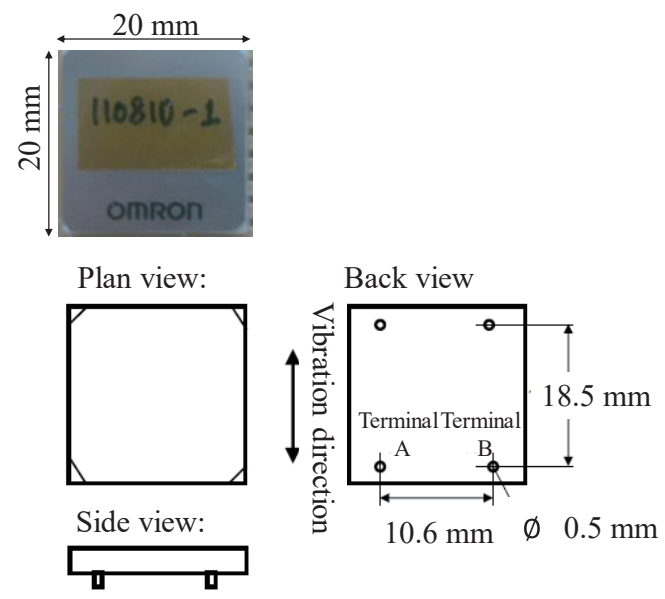

Fig. 8. (Color online) Shape and size of the developed sensor.

Table 2

Parameters used in the developed sensor.

(a) Parameters of mechanical part

\begin{tabular}{lc}
\hline Mass of electret oscillator $(\mathrm{g})$ & 0.0018 \\
Natural frequency $(\mathrm{Hz})$ & 8.8 \\
Quality & 19.4 \\
\hline (b) Parameters of electrode part & \\
\hline Gap between electrodes $(\mu \mathrm{m})$ & 70 \\
Thickness of electrets $(\mu \mathrm{m})$ & 15 \\
Relative permittivity & 2.1 \\
Resistance $(\mathrm{M} \Omega)$ & 1 \\
Charge density of surface $\left(\mathrm{C} / \mathrm{m}^{2}\right)$ & $0.7 \times 10^{-3}$ \\
\hline
\end{tabular}

(c) Dimensions of the sensor

Width of electret oscillator $(\mathrm{m})$

Length of electret oscillator $(\mathrm{m})$

0.018

Width of electret electrode $(\mu \mathrm{m})$

Gap between electret electrodes $(\mu \mathrm{m})$

Number of electret electrodes 


\subsection{Generated power waveform}

Vibration tests were performed at different frequencies and different velocities. To generate different velocities, different amplitudes were applied in the vibration tests. Figure 9 shows an example of the time series of vibration velocity and the generated power expressed in volts obtained in the constant amplitude vibration test with an $11 \mathrm{~Hz}$ vibration frequency and $0.15 \mathrm{~mm}$ amplitude of vibration. The testing conditions of constant amplitude vibration tests will be explained in the next section in more detail. The vibration velocity was calculated from the measured displacement using the laser displacement measurement system. The time series shown in Fig. 9 are the results of low-pass filtering of $20 \mathrm{~Hz}$ to obtain smooth curves. In Fig. 9, a phase difference between the vibration velocity and the measured voltage is seen, but the waveform is smooth with no ripple shapes.

After adjusting the phase shift between the waveforms of vibration velocity and the measured voltage, the ratio of the two values was determined to obtain Fig. 10. The ratio is around 1.00 for all the points in the waveform, and the waveforms of vibration velocity and the measured voltage can be considered to be almost the same on the basis of Fig. 10.

\subsection{Frequency characteristics}

In order to clarify the relationships among vibration frequency, vibration velocity, and generated power expressed in volts, in this study, constant-amplitude vibration tests of the developed sensor were conducted using a vibration table. Figure 11 shows the testing setup. The generated voltage time series were measured and used to obtain the voltage amplitude and phase difference at different velocities and frequencies. The generated voltage of the sensor was measured with a data logger in the voltage range of $1 \mathrm{~V}$ and $\mathrm{A} / \mathrm{D}$ conversion resolution of 16 bits. The sampling rate was set to be $10 \mathrm{kHz}$.

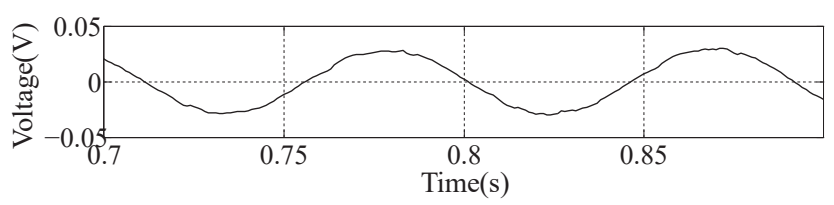

(a)

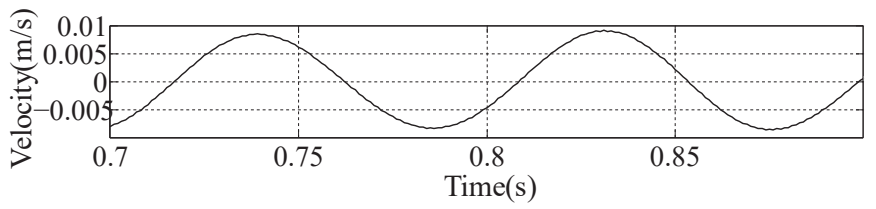

(b)

Fig. 9. Waveform in the developed sensor. (a) Generated voltage. (b) Vibration velocity.

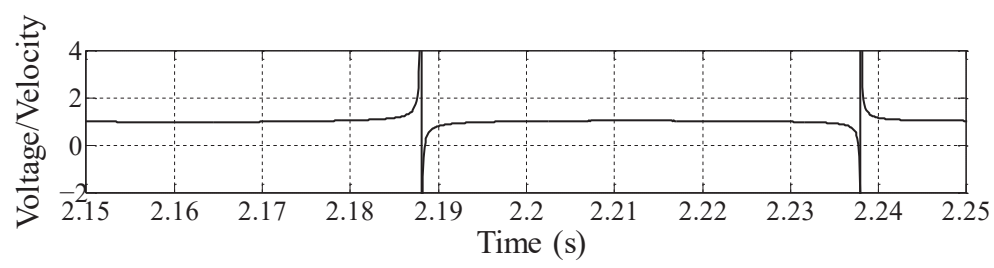

Fig. 10. Ratio of generated voltage to vibration velocity. 


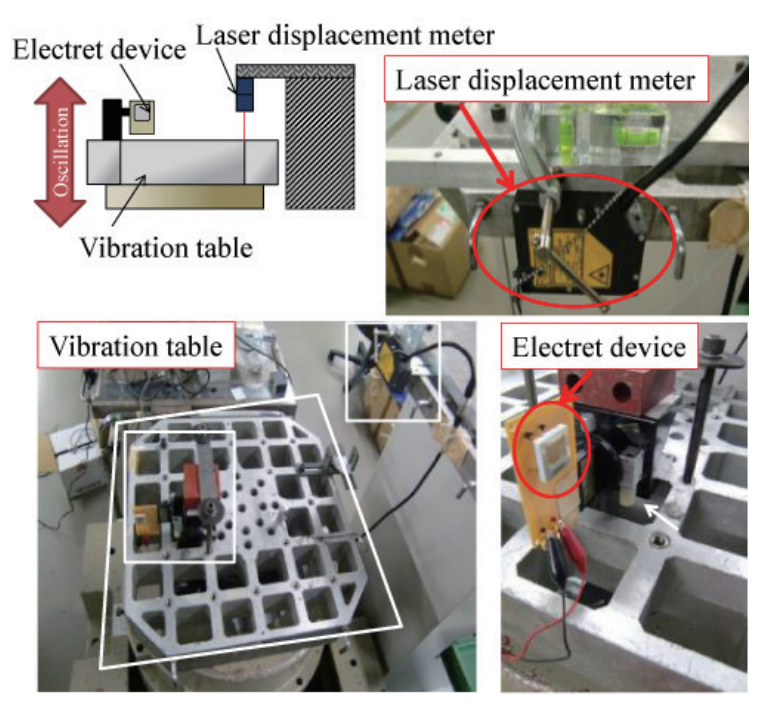

Fig. 11. (Color online) Vibration test setup.

Figure 12 shows the test results with the frequency of $11 \mathrm{~Hz}$. This figure shows the relationship between the vibration velocity and the generated voltage. The velocity was changed by using different vibration amplitudes in the same frequency. From this figure, a clear linear relationship between the vibration velocity and the generated voltage amplitude can be observed. The linear relationship between the vibration velocity and the generated voltage amplitude was confirmed for all vibration frequencies. The slope of the line in the relationship is constant in a specific vibration frequency, but it differs with different frequencies. The slope of the line means the generated voltage amplitude per unit velocity (i.e., $1 \mathrm{~m} / \mathrm{s}$ ), and it also can be considered as the coefficient showing the relationship between the vibration velocity and the generated voltage amplitude at a specific vibration frequency. Therefore, in this study, the slope of the line is called as the transfer function between the vibration velocity and the generated voltage, and will be used in the random amplitude vibration measurements in the following part of this study.

Figure 13 shows the transfer function and phase obtained in the tests under vibration at different frequencies ranging from 1.5 to $20 \mathrm{~Hz}$. In the figure, the curves obtained in the numerical analysis are also shown. A peak can be found at the resonance frequency of the sensor. As for the phase, differences between the measured values and the analyzed values can be seen. The cause of this discrepancy has not been clarified, but it can be considered that the sensor characteristics will be tested and provided by the suppliers in the actual application of the sensor, so in this study, the characteristics obtained in the tests were utilized. On the other hand, the transfer function obtained in the tests coincides with that in numerical results. From these results, the numerical simulation can be used to predict the transfer function of the electret vibration sensors.

\subsection{Application to random vibrations}

The purpose of this study is to propose a new sensor for bridge monitoring. In actual bridges, random vibrations always occur due to the passage of vehicles or other causes. In this study, to simulate such situation, random vibration tests using a steel girder model were conducted to 


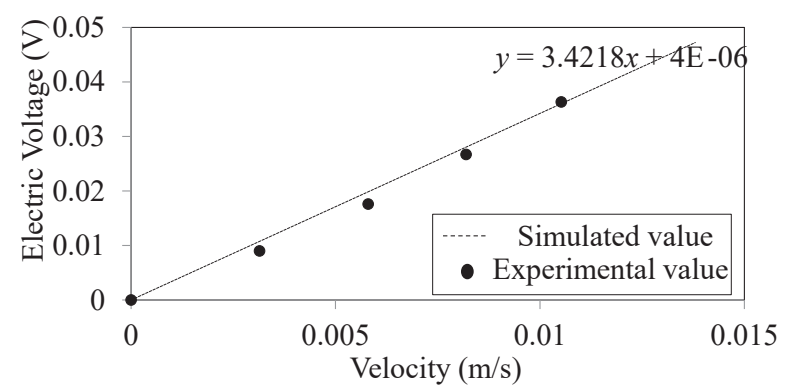

Fig. 12. Relationship between generated voltage and vibration velocity $(11 \mathrm{~Hz})$.

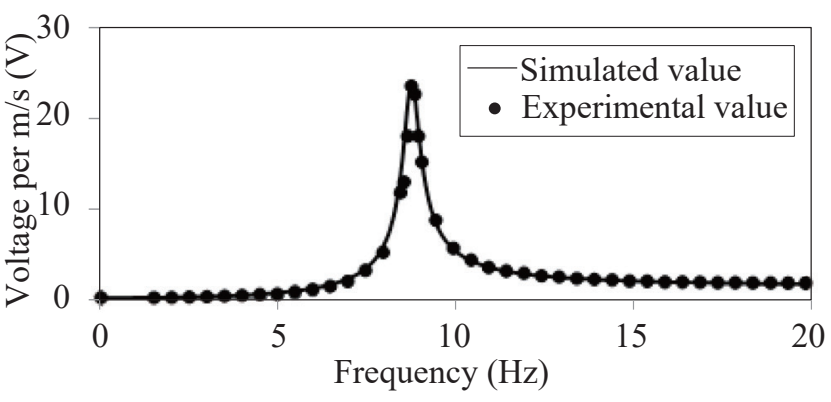

(a)

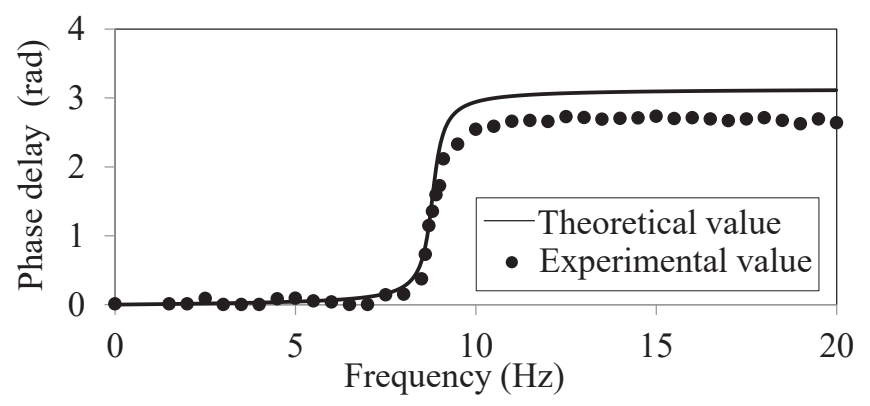

(b)

Fig. 13. Frequency characteristics of the developed sensor. (a) Transfer function. (b) Phase.

investigate the applicability of vibration measurements using the proposed sensor. In these tests, the possibility that by using the transfer function and the phase properties, the velocity under random vibration can be evaluated was discussed.

Figure 14 shows the test setup for the random vibration tests. A hydraulic dynamic actuator was used to generate random vibration on the steel girder model by applying cyclic loads at the center of the girder span. The developed vibration sensor with an electret, a 3-axis acceleration sensor, and a laser displacement measurement system was put on the girder at the center of the bending span. The input signal for the vibration was created by the random vibration at a frequency of less than 10 $\mathrm{Hz}$ considering typical bridge characteristics. The same measurement system as described in Sect. 3.2 was used to measure the data during the tests.

Figure 15 shows the velocity calculated from the generated voltage of the electret vibration sensor and that obtained from displacement. The conversion method from the generated voltage to vibration velocity in random vibration utilizes the whole frequency range of the transfer function. To calculate the vibration velocity time series, the time windows and overlap-add method were used. ${ }^{(18)}$ The two evaluated velocities agree well with each other. Therefore, the developed sensor is promising for evaluating velocity under random vibration as in actual bridge situations.

In actual bridges, environmental conditions such as temperature can affect the bridge characteristics including natural frequency, but the vibration velocity can be calculated even when the frequency of a bridge changes, because the conversion from the generated voltage to vibration 


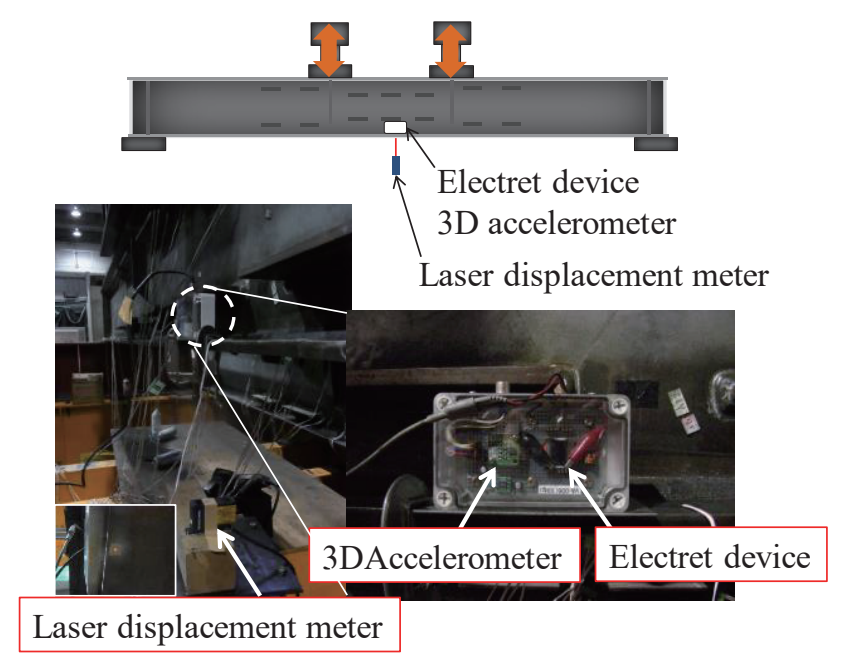

Fig. 14. (Color online) Random vibration test setup.

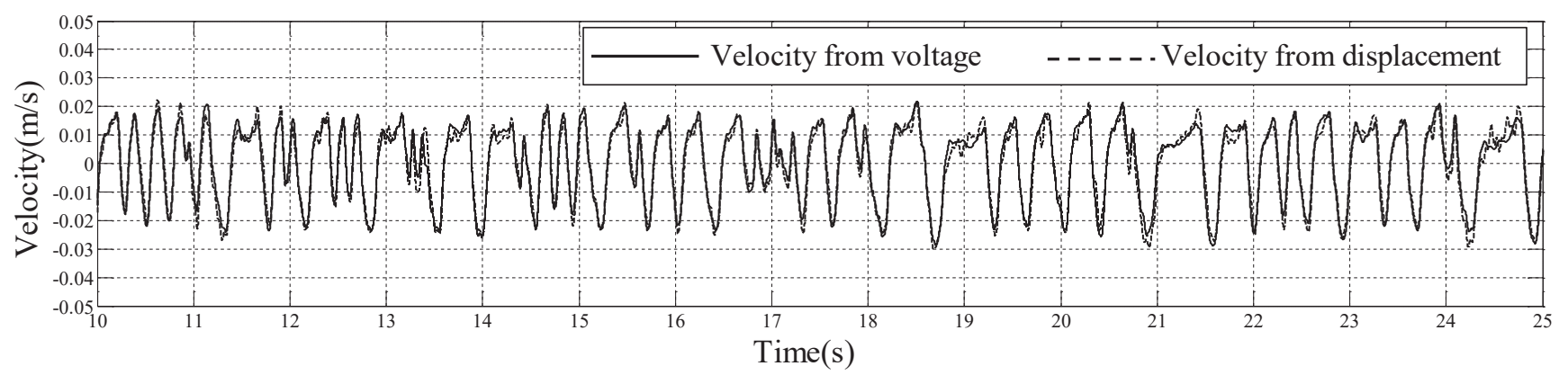

Fig. 15. Evaluated and measured velocities.

velocity uses the whole frequency range of the transfer function. The proposed sensor system has the robustness for the characteristic changes.

\section{Development of Vibration Sensors with Different Resonance Frequencies}

In bridge monitoring, one of the most important purposes is to understand the vehicle-induced displacement. In particular, in steel bridges, in order to identify the causes of displacement-induced fatigue cracks, the displacement of the components needs to be obtained. However, vehicleinduced vibration has low-frequency components of $0.1 \mathrm{~Hz}$ order in many cases. In the previous study, some difficulty was encountered in measuring the low-frequency vibration because the power generation level decreases with increasing difference from the resonance frequency. Up to now, it has been difficult to develop the vibration sensors with an electret electrode for lower frequencies owing to the difficulty in obtaining the appropriate spring stiffness in the vibration sensor for lower frequencies. In addition, it can also be important in some cases to cover a wider frequency range, for example, by utilizing several sensors with different resonance frequencies. 
In this study, new vibration sensors with the resonance frequencies of 3, 13.3, and $29.5 \mathrm{~Hz}$ were developed. The sensors with the resonance frequencies of 13.3 and $29.5 \mathrm{~Hz}$ have the same structure as the original prototype sensor with an $8.8 \mathrm{~Hz}$ resonance frequency, but the sensor with the resonance frequency of $3 \mathrm{~Hz}$ has a longer spring than the others, as shown in Fig. 16. The development of the sensor with the resonance frequency of $3 \mathrm{~Hz}$ is especially challenging, and hence it is necessary to evaluate the performance by numerical simulation and vibration tests.

\subsection{Numerical simulation}

The numerical simulation of the newly developed three vibration sensors was conducted to predict their power generation levels. The same procedure as explained in the previous section was used in the numerical simulation. The parameters used in the numerical simulation are listed in Table 3. Figure 17 shows the numerical simulation results in the form of the transfer function for gain and phase. Peaks in the gain that correspond to the generated power are clearly observed at each respective of resonance frequency as shown in Fig. 17(a). From Fig. 17(b), the phase change characteristics are confirmed. The phase change occurs at the resonance frequency.

\subsection{Vibration tests to evaluate the transfer function}

Vibration tests were conducted to evaluate the transfer function of the developed three sensors. In the tests, the designed input signal considering the combination of sweep and random wave components was used unlike in the previous study in which constant-amplitude vibrations were used. This designed input signal can cover low to high frequencies. The designed input signal shown in Fig. 18 was applied.

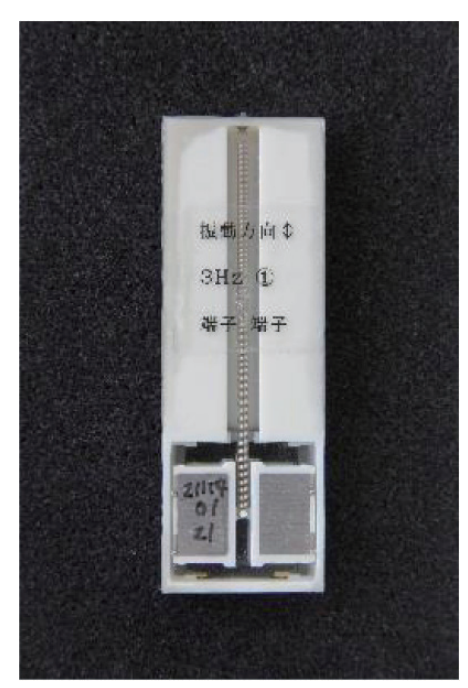

Fig. 16. (Color online) Shape of the sensor with $3 \mathrm{~Hz}$ resonance frequency. 
Table 3

Parameters used in the developed sensor.

(a) Parameters of mechanical part

\begin{tabular}{lccc}
\hline Sensor & $3.0 \mathrm{~Hz}$ & $13.3 \mathrm{~Hz}$ & $29.5 \mathrm{~Hz}$ \\
\hline Mass of electret oscillator $(\mathrm{g})$ & 3.6 & 3.6 & 3.6 \\
Natural frequency $(\mathrm{Hz})$ & 3.0 & 13.3 & 29.5 \\
Quality factor & 7.9 & 150 & 150
\end{tabular}

(b) Parameters of electrode part

\begin{tabular}{lccc}
\hline Sensor & $3.0 \mathrm{~Hz}$ & $13.3 \mathrm{~Hz}$ & $29.5 \mathrm{~Hz}$ \\
\hline Gap between electrodes $(\mu \mathrm{m})$ & 70 & 70 & 70 \\
Thickness of electrets $(\mu \mathrm{m})$ & 15 & 15 & 15 \\
Relative permittivity & 2.1 & 2.1 & 2.1 \\
Resistance $(\mathrm{G} \Omega)$ & 1 & 1 & 1 \\
Charge density of surface $\left(\mathrm{C} / \mathrm{m}^{2}\right)$ & -0.000826 & -0.000826 & -0.000826 \\
Surface $(\mathrm{V})$ & -726 & -700 & -700
\end{tabular}

(c) Dimensions of the sensor

\begin{tabular}{lccc}
\hline Sensor & $3.0 \mathrm{~Hz}$ & $13.3 \mathrm{~Hz}$ & $29.5 \mathrm{~Hz}$ \\
\hline Width of electret oscillator $(\mathrm{m})$ & 0.020 & 0.020 & 0.020 \\
Length of electret oscillator $(\mathrm{m})$ & 0.0135 & 0.0135 & 0.0135 \\
Width of electret electrode $(\mu \mathrm{m})$ & 7000 & 7000 & 7000 \\
\hline
\end{tabular}

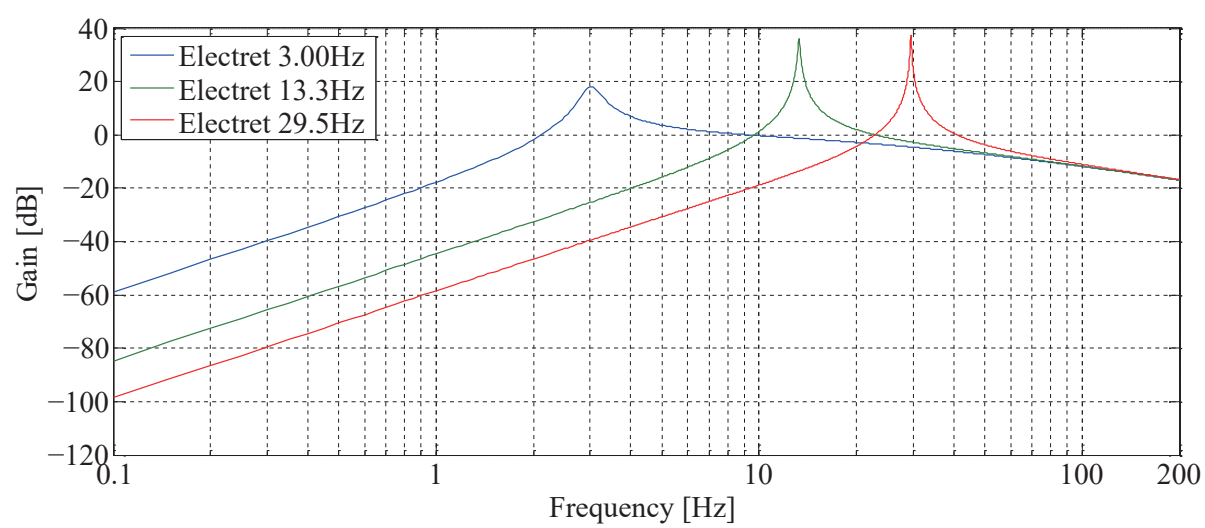

(a)

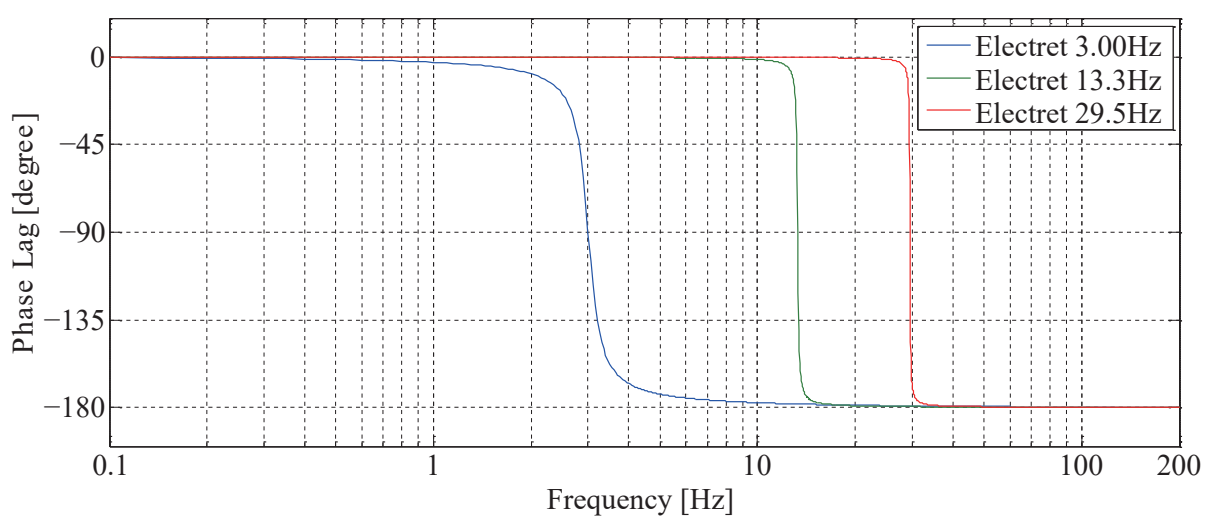

(b)

Fig. 17. (Color online) Numerical simulation results of developed sensors. (a) Transfer function. (b) Phase. 


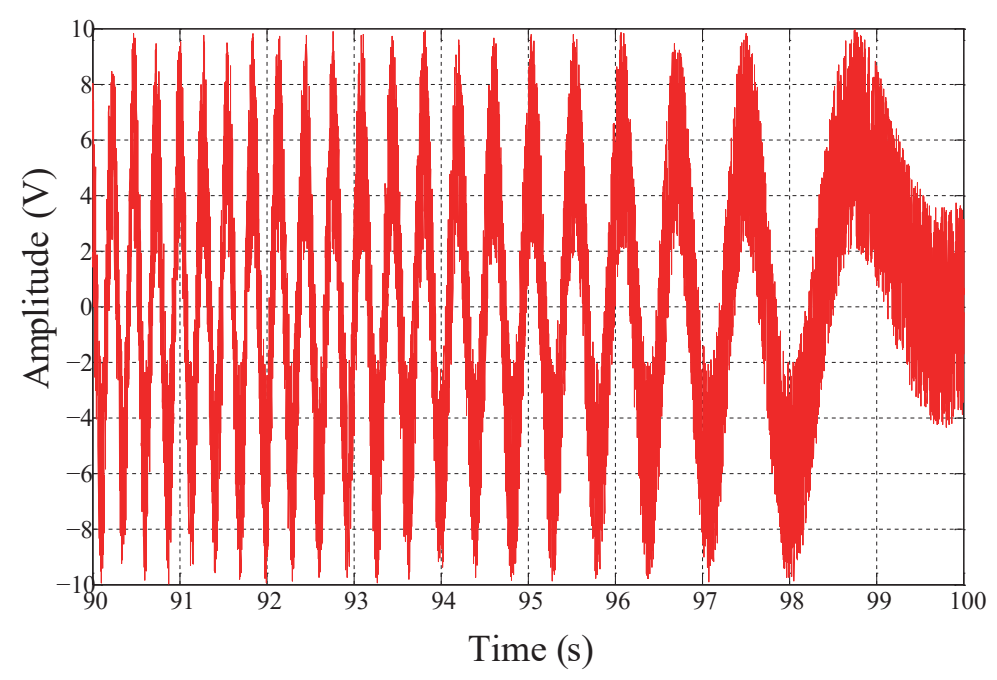

Fig. 18. (Color online) Designed input signal.

In the vibration tests, a dynamic testing machine was used and the test setup is shown in Fig. 19. Displacement data can be obtained directly from the testing machine to the data logger used in the tests. Displacement data are used to calculate vibration velocity. The data logger also measured the generated voltage of the sensors.

Figure 20 shows the transfer function and the phase (lag). Similar features can be found in the numerical results and the vibration test results. Peaks in the gain are clearly observed at each respective resonance frequency as shown in Fig. 20(a). In Fig. 20(b), the phase change characteristics of the resonance frequency can be seen.

\section{An Attempt to Measure Actual Bridge Vibration Using Developed Sensors}

In order to discuss the applicability and possible problems of the newly developed sensors, vibration measurements of an actual bridge were attempted (Fig. 21). The target bridge is a truss bridge spanning a river. Because of the deterioration of the bridge, the maximum weight of vehicles is limited to $5.5 \mathrm{t}$. Therefore, the vibration energy is considered to be relatively small in this bridge. The measurement is performed at the location near the supports of the bridge as shown in Fig. 21.

The three newly developed sensors with the resonance frequencies of 3, 13.3, and $29.5 \mathrm{~Hz}$ were fixed to the lower flange of a transverse girder of the bridge together with a laser displacement meter and acceleration sensors at the same location. The measurement setup is shown in Fig. 22.

Figure 23 shows an example of the measured generated voltage of the three sensors. The sensor with the $3 \mathrm{~Hz}$ resonance frequency generated much more power than the other sensors owing to the low-frequency vibration of the bridge component and the small vibration amplitude. Therefore, this time, the data of the sensor with the $3 \mathrm{~Hz}$ resonance frequency are used in the following part.

Figure 24 shows an example of the measured acceleration, velocity, and displacement and those evaluated vibration information using the developed sensors. Similar waveforms of the measured values and the evaluated values can be seen in each of the figures, especially in displacement and 


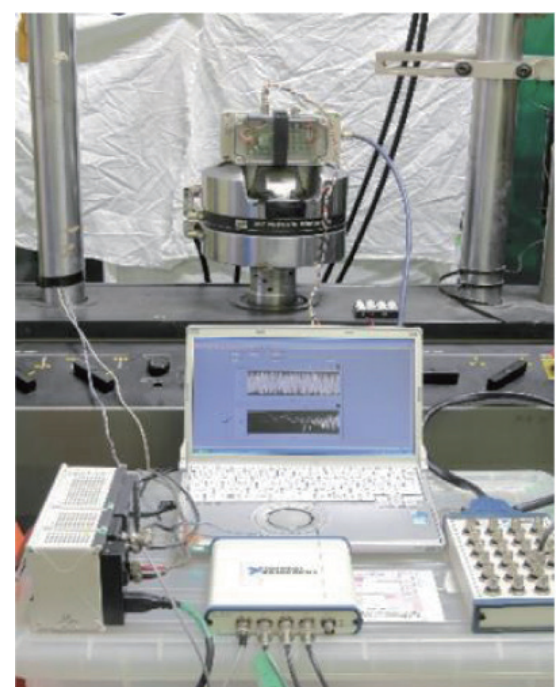

Fig. 19. (Color online) Vibration test setup for developed sensors.

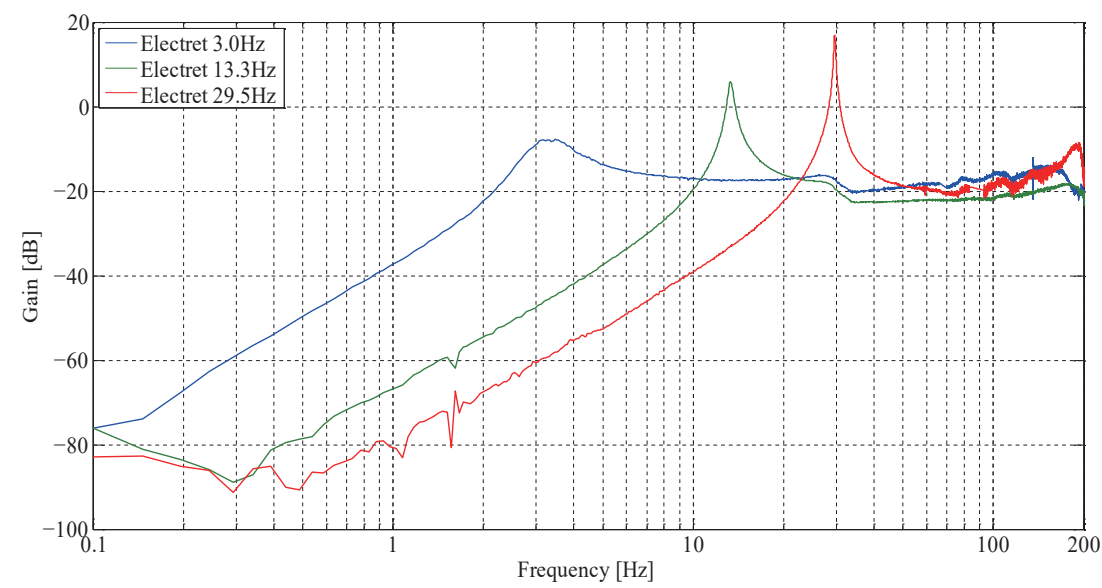

(a)

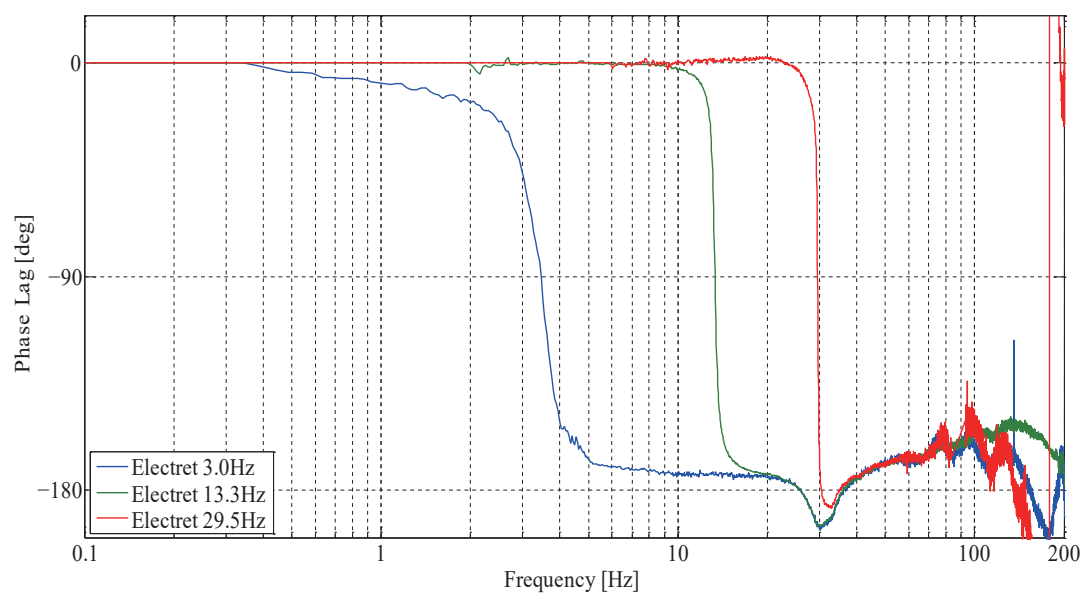

(b)

Fig. 20. (Color online) Frequency characteristics of developed sensors. (a) Transfer function. (b) Phase. 


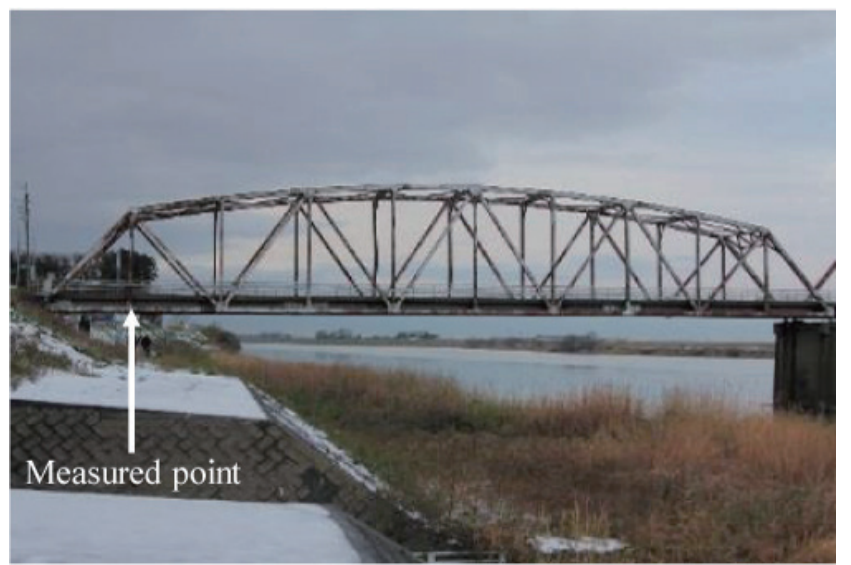

Fig. 21. (Color online) Target truss bridge.

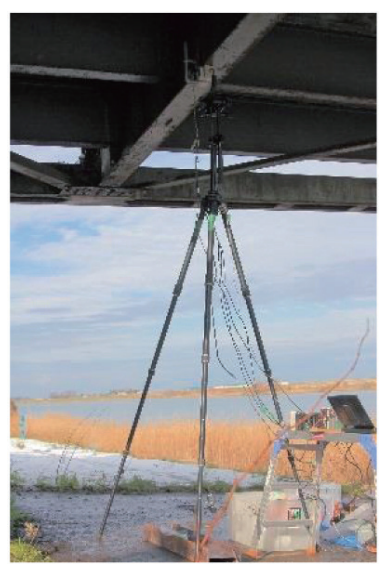

(a)

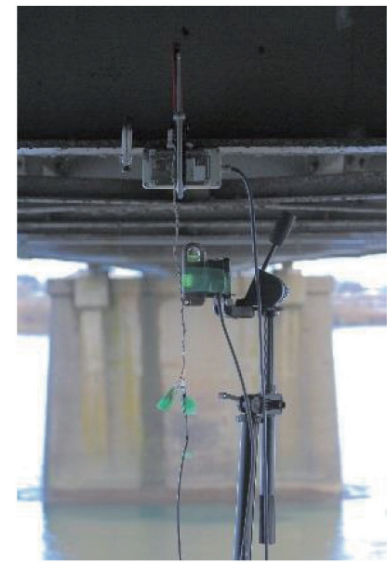

(b)

Fig. 22. (Color online) Measurement setup. (a) Laser displacement meter. (b) Electret vibration sensors (upper) and laser displacement meter (lower).

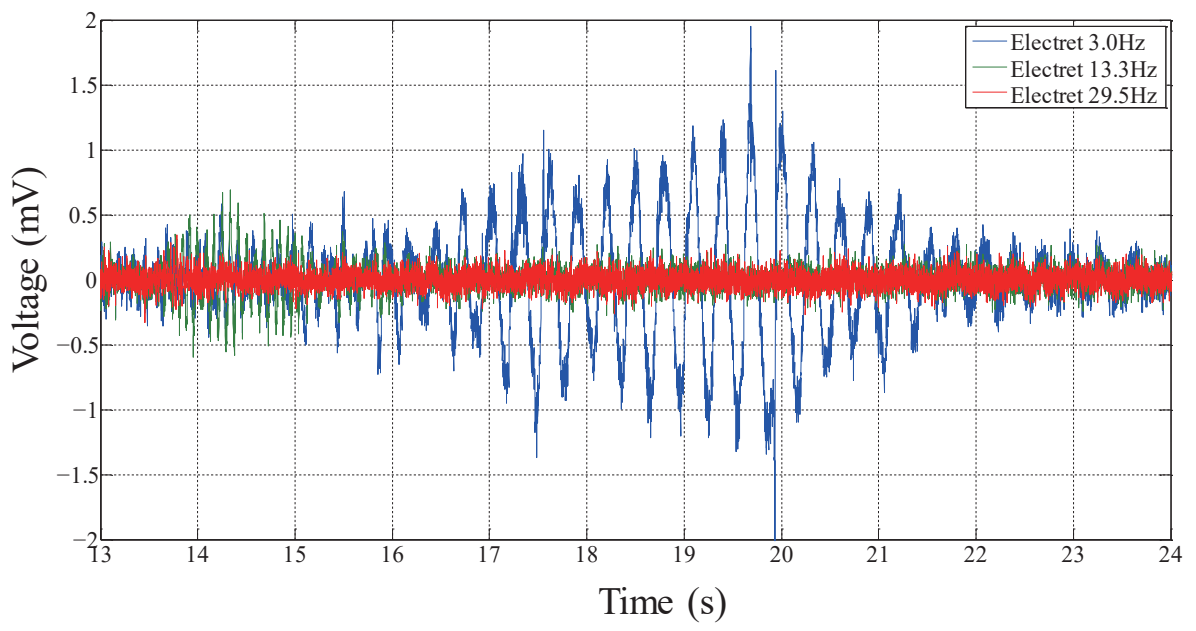

Fig. 23. (Color online) Example of measured data of generated voltage. 


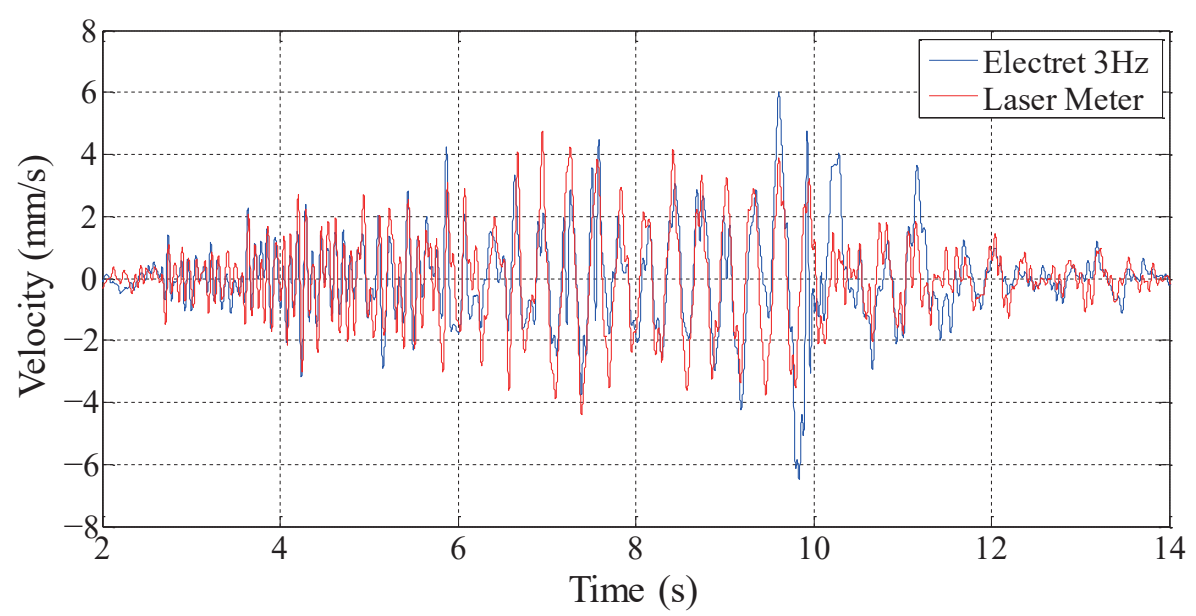

(a)

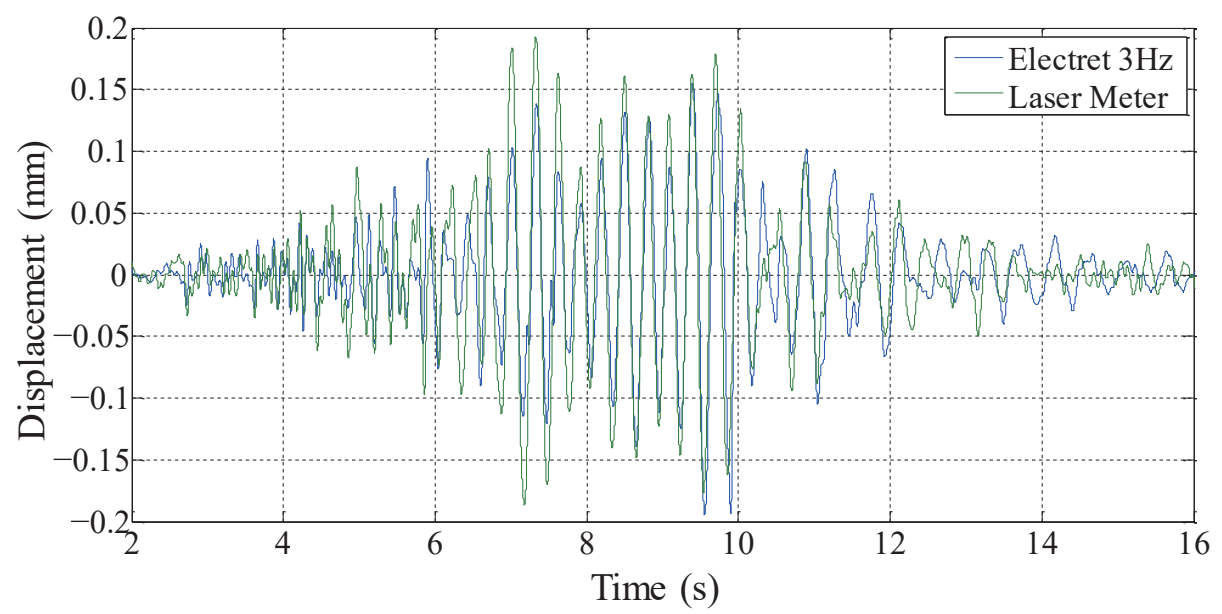

(b)

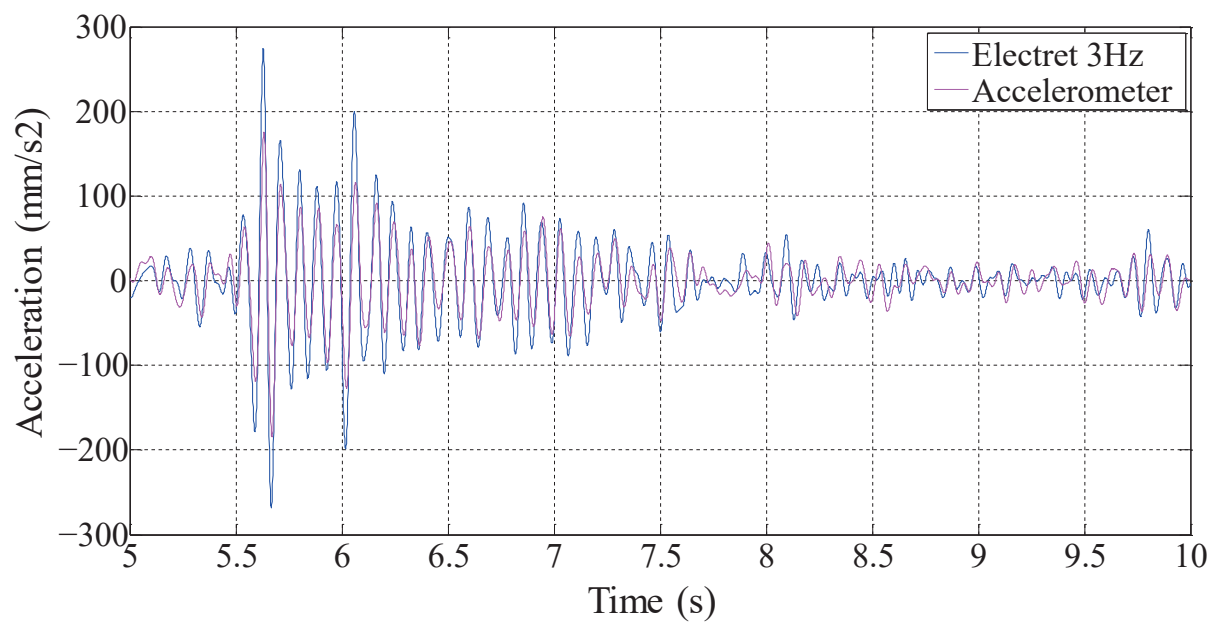

(c)

Fig. 24. (Color online) Measured and evaluated values. (a) Velocity. (b) Displacement. (c) Acceleration. 
acceleration, but the evaluated amplitudes of the waveforms showed difference from the measured values. Some improvement in evaluation may be needed in cases with smaller vibration amplitude. The sensors will be applied to other situations to elucidate the applicable conditions.

\section{Conclusions}

In the structural monitoring of bridges, it is important to obtain information on the vibration characteristics and deformation shape of components. In this study, innovative vibration sensors based on the energy-harvesting concept were investigated using electret material. The concept and fundamental characteristics of the power generation sensors were described. Furthermore, in this study, the development of a new vibration sensor with an electret with much lower resonance frequency was undertaken, and the performance evaluation was conducted for the new sensor. The conclusions of this study can be listed as follows.

a) An appropriate electret electrode structure for a vibration sensor was proposed on the basis of the numerical analysis results.

b) The generated power expressed in volts in the proposed sensor is proportional to vibration velocity with a constant slope for a specific frequency. The constant slope is called the transfer function from the generated voltage and vibration velocity. The transfer function can be estimated accurately by numerical simulation, so in the design of the sensors, the numerical simulation can be utilized.

c) New vibration sensors with lower resonance frequencies were developed. Among them, the vibration sensor of $3 \mathrm{~Hz}$ has a special dimension with a larger length using a long spring. The characteristics of the sensors were evaluated by numerical simulation and vibration tests.

d) Vibration was measured using the newly developed sensors on an actual bridge, and the obtained velocity, displacement, and acceleration from the generated power of the developed sensors were compared with the measured values. Similar waveforms of the measured values and the evaluated values can be seen in each of the figures, especially in displacement and acceleration, but the evaluated amplitudes of the waveforms showed differences from the measured values. The causes of scattering in the values evaluated from the generated voltage of the newly developed sensor will be investigated by clarifying the applicable conditions.

In this study, the development of the newly proposed vibration sensor using electret power generation phenomenon was shown. The advantages of the proposed vibration sensors are in their easy installation to structures and in the evaluation of vibration velocities and displacements without power supply to the sensors and any fix points to obtain displacement information.

In bridge monitoring, environmental conditions such as temperature can affect the structural behaviors and measurement results. However, the changes in the structural behaviors of bridges can be measured using the proposed sensors, because the conversion from the generated voltage to velocity is performed using the whole range of frequency, but the long-term measurement using the proposed sensors should be conducted in the future to examine their applicability to various conditions.

\section{References}

1 Y. Kobayashi, C. Miki, and A. Tanabe: J. JSCE 773 (2004) 99 (in Japanese).

2 Y. Kobayashi, C. Miki, and A. Tanabe: J. JSCE A 62 (2006) 794 (in Japanese).

3 T. Shimozato, J. Murakoshi, Y. Tamakoshi, and M. Takahashi: Bridge Found. 43 (2009) 55. 
4 T. Yoshioka, M. Harada, H. Yamaguchi, and S. Ito: J. Struct. Eng. A 54 (2008) 199.

5 T. Yoshioka, H. Yamaguchi, S. Ito, and M. Harada: J. Struct. Eng. A 55 (2009) 295.

6 C. Miki, H. Yamaguchi, T. Hanji, and A. Tanabe: J. Struct. Eng. A 56 (2010) 671.

7 K. Takahashi, S. Naito, M. Seki, A. Ichikawa, and C. Miki: J. JSCE A 64 (2008) 235 (in Japanese).

8 T. Miyashita, H. Ishii, Y. Fujino, T. Shoji, and M. Seki: J. JSCE A 63 (2007) 277 (in Japanese).

9 S. Kim, S. Pakzad, D. Culler, J. Demmel, G. Fenves, S. Glaser, and M. Turon: Int. Conf. Information Processing in Sensor Networks (2007) p. 254.

10 S. Jang, H. Jo, S. Cho, K. Mechitov, J. A. Rice, S. H. Sim, H. J. Jung, C. B. Yun, B. F. Spencer, Jr., and G. Agha: Smart Struct. Syst. 6 (2010) 439.

11 K. Park, S. Kim, H. Park, and K. Lee: Eng. Struct. 27 (2005) 371.

12 H. Sekiya, K. Kimura, and C. Miki: Sensors 16 (2016) 257.

13 H. Okamoto and H. Kuwano: Funct. Mater. 30 (2010) 7.

14 Y. Kobayashi: Bridge Found. 44 (2010) 69.

15 K. Takeya, E. Sasaki, and Y. Kobayashi: J. Sound Vibr. 361 (2016) 50.

16 M. Doi and M. Oba: Funct. Mater. 30 (2010) 21.

17 K. Matsumoto, K. Saruwatari, and Y. Suzuki: IEEJ Trans. Electronics, Information and Systems 132 (2012) 344.

18 H. Yamaguchi, E. Sasaki, and G. V. Minesawa: J. JSCE A 70 (2014) 150 (in Japanese).

19 T. Takamatsu: Proc. 7th Int. Symp. Electrets (1991) p. 106.

20 Y. Suzuki, D. Miki, M. Edamoto, and M. Honzumi: J. Micromech. Microeng. 20 (2010) 104002. 